THEORIA ET HISTORIA SCIENTIARUM, VOL. VII, N ${ }^{\circ} 2$

Ed. Nicolas Copernicus University 2003

H. John Caulfield* , Stephen W. Kercel**

\title{
Unconscious perception and communication
}

\begin{abstract}
In the development of life and mind on earth, consciousness seems to have emerged rather late in the process. Some researchers believe animals as primitive as salamanders have some rudimentary consciousness. However, consciousness appears to be volitional. Humans can perform many functions unconsciously, while being conscious of very little of what is happening to them and in their world. An attention function brings information to our conscious process. We suggest that genuine communications with manmade artifacts, a technological feat not yet achieved, would need to go through a very similar attentional process.
\end{abstract}

\section{Introduction}

The roles and even the realities of unconscious and conscious mental activity are still disputed among cognitive scientists, philosophers, brain experts, and the like (Korzybski, 2000; Dilts, 1983). We offer here a very simple explanation of how these two activities may contribute to decision making - the brain's primary function in any evolutionary interpretation. Then, we seek to use that insight to offer advice on how to make the best Human-Computer Interface - HCI.

According to this explanation, a mind makes continuously updated hypothetical models of the body and its environment. In fact, abduction, or the

* Address for correspondence: Fisk University, 1000 17th Ave., N. Nashville, TN 37208, USA. Email: hjc@fisk.edu

** Address for Correspondence: 2 Brian Drive, Brunswick ME 04011, USA. Email: kercell@suscom-maine.net 
process of constructing appropriate hypotheses with better than random success, is one behavior of living minds do that no man-made artifact has ever performed (Fodor, 2000) Then (especially if the already-abducted hypotheses appear to be utterly inconsistent with the incoming sensory data) an attentional filter chooses which, if any, of those models will be brought into the conscious process so that they might be updated.

The abstraction of multiple streams of sensations into percepts occurs at an unconscious level (Freeman, 1999) The occasional decision to refer percepts to a higher level is based on a unconscious perception that something does not feel right (Damasio, 1999; Sacks, 1995) or, alternatively, something important has just happened. This concept is far from new. What is new is the justification as to why the organization of the brain might have evolved this way, and a collection of verifiable predictions resulting from that concept.

This explanation is justified in three ways. First, it is a reasonable engineering design, given the slow speed and vast parallelism of the known neurochemical processing that nature must use. Second, it is seen as an easy way to predict correctly human conscious experiences in many, quite-diverse circumstances. Third, it makes a great deal of sense in terms of evolution. That is, we can use these considerations to show how consciousness may have evolved, and what role it would play. While these arguments do not prove this explanation to be correct, they do lend it credence.

Most of the brain and especially the cerebellum, seem to be concerned with updating their hypothesized models of the body and of the world of which it is a part. This truly monumental goal is attacked using the multi-level self-referential formal processes that only brains have so far accomplished. Indeed it may be that when confronted with ambiguities and uncertainties, the brain may model the various likely possibilities independently. It will also seek confirming and disconfirming data to resolve those ambiguities (Kovacs, Papathomas, Yang and Feher, 1996)

For conscious control to be effective, it must work as fast as the threats and opportunities in the world change. However, consciousness operates very slowly. Humans require almost half a second to become conscious of something (Libet, Freeman, and Sutherland, 1999). Yet the firing rate for neurons is only about one millionth that of inexpensive modem computers. To facilitate conscious behavior in 'real time,' we suggest that the brain uses an attention process to simplify the task. Attention selects what will become conscious and reinterprets ambiguous perceptual information by abducting a more general hypothetical model that accommodates all the percepts without ambiguity. For example, given the Necker cube we can observe the resolution of competing hypotheses in action. By an act of volition, we can interpret the visual sensation as the image of a cube coming 'out of the page'. By another act of volition, we can interpret the visual 
sensation as the image of a cube going 'into the page'. From the combination of visual and tactile experiences with semi-transparent cubes, we can imagine either hypothesis as being consistent with the visual data.

However, we have more sensations available, and can make a broader hypothesis. If we touch the page, we note that the image is flat, without the detectable edges of a 3dimensional cube. If we flip the page, we note that the image has no thickness. A more general and more satisfying hypothesis emerges; the object is not a cube at all. It is a flat object with a superficial resemblance to several different kinds of cube. In fact, once we've integrated that hypothesis into our world model, we must deliberately choose to ignore the tactile part of the data to appreciate why we once interpreted the image as a cube. Conscious experience becomes clear, unitary, and distinct. Ambiguity may be inferred logically but not consciously experienced except by psychotics, who find the experience to be terrifying. An ambiguous figure may lead to alternating perceptions but only one at a time. Thus we become conscious of a small fraction of what is in the unconscious at any instant and even what we do experience consciously is filtered to remove ambiguities and prevent information overload.

Another way of saying this is only analogous, but many computer-oriented people understand it well. Many mental processes in occur in parallel - often a single conscious perception or thought draws from activities in multiple areas of the brain. But an executive function such as consciousness typically does not function in parallel. It is there to direct either learning, or action, or both. None of those should be multiple. The brain needs some way to focus consciousness on what is most important at any instant. This role of attention is selection of which mind-brain functions should be coupled into consciousness. By working out simple consequences of the hypothesis just outlined, we can predict aspects of our conscious and unconscious mental activities, which can be tested readily. We have collected data that seem to confirm the predictions and thus support the hypothesis. This combination of empirical support and plausible evolutionary explanation suggests that this interpretation of the roles of the conscious and unconscious minds may be substantially correct.

To restate our model as succinctly as possible:

1) The brain (mostly the cerebellum) abducts an ongoing, continually updated, unconscious model of the body and its world using many parallel functional components that sometimes do and sometimes do not correspond to specialized regions of the brain.

2) When, because of ambiguous or unfamiliar or incompletely understood data, there are multiple plausible interpretations of the world, the brain abducts a larger model to accommodate them and actively directs the sensory system to help resolve the ambiguities. 
3) The content of consciousness is provided from these unconscious percepts by selection (not everything in a complex situation of a complex body can be conscious at once) and disambiguation (only one interpretation can be conscious at any time even if the situation is ambiguous). This process or brain functionality is called 'attention'.

\section{Why this model makes evolutionary sense}

Why would an organism do any mental processing at all? Clearly, one of the answers is to decide what to do next. Model-based control is widely used to tell a mechanism what to do next, because it is fast and efficient in use of computational resources. Artificial neural networks are useful at making models, although they offer no warning as to where they might fail. That nature might use neural models to serve as part of a model based control scheme in organisms seems at least reasonable. 'The basis for the experience of consciousness is the symbolic representation of the world and of the individual to that individual's brain.' (Creutzfeldt, 1979). But our body and our world are so complex and rapidly- changing that serial processing at even the crudest level with the brain's slow electrochemical computer is impossible. Parallelism is essential. However, the parallelism must be of quite a different character than 'massively parallel architectures' and the consequent combinatorial explosion of interprocess communications in contemporary computers (Aiello and Bach-Y-Rita, 2000).

Another problem nature faced is that while updating a model (in the spirit of a Kalman filter) may be done quickly, constructing a model $a b$ initio is far too inefficient and slow for a complex body in a complex world. It seems more likely that nature would develop and exploit a system non-local in space-time to keep a great number of body parts and world components modeled and available to be abstracted into percepts available to consciousness as needed. Attention and consciousness operate at two different levels. The attention process detects ambiguity and apprises the conscious process of its presence. Consciousness abducts a new hypothesis to resolve the ambiguity.

There is no largest model of a complex system, be it the world, or a human being (Rosen, 1991). In practical terms, a multi-level self-referential natural system \{endogenous system) has infinitely many behaviors that are consistent with its causal entailments. A multi-level self-referential formal system (impredicative system) has infinitely many behaviors that are consistent with its inferential entailments (Kercel, 2001). Thus, it is possible for the world or the human himself to exhibit a behavior not anticipated by the human's internal models. Although the human can never construct a largest model, he can use the 
results of a bizarre occurrence to abduct a better hypothesis and enlarge his internal models. It has long been recognized that abstraction of meaning from a stream of signs is done by a non-local process in the brain (Bateson, 1972). It should be not surprising if abduction, a similar non-local process should function by organizing itself across a substrate many including simple elements.

Notice how this departs from mechanistic processes. Mental processes are value laden. The modeller constructs a better model to move toward some internally chosen goal. The role of consciousness seems to be to plan and direct actions that will move the model body toward its goal in its model world.

As evolution is very conservative, it can be expected to use a good modelprocessing method over and over once it is discovered. So the attention's selection of percepts to enter consciousness for broadening of the hypotheses in our models is likely to be used in other mental activities such as personality, invention, and even laughter.

In the conclusions of this paper, we offer a discussion of how such a conscious control might have evolved in a world previously devoid of consciousness. But first, we want to explore what predictions seem to flow from this simple analysis and seek evidence as to whether those predictions hold for the world of conscious animals.

\section{Predictions on perception}

If we assume the model whose plausibility we have just argued, what can we predict about what a creature using such a method would perceive? Remember, of course, that the only way the brain can perceive something distant from it is to model the distant situation, abstracting sensation into percepts, and unconsciously reacting at an emotional level, of 'feeling' the percepts. When the feeling actuates the attention process, is brings the situation to our conscious process. We experience the world through the model that we construct of it. The effect can be quite dramatic; we see so vividly the person behind the mirror who should have been there to account for what our eyes detected if light had behaved as normal - traveling a straight line from the object to our eyes.

The most obvious prediction is that we will never directly perceive an ambiguous situation in the world even when presented with one. People suffering from psychosis do not see one distinct situation or alternate among distinct situations. They experience contradictory interpretations of the world simultaneously. The second prediction is that we should be able to switch attention very quickly among models of body parts - much faster than we could possibly build up such models $a b$ initio. 


\section{Predictions on mental functioning}

According to this model, your unconscious brain can construct models without those models coming to attention. In a symbol-using creature like man, the unconscious may well model problems, a means to judge the suitability of solutions, and a multiplicity of potential solutions. These are the minimal requirements for an evolutionary approach to problem solving. Good solutions can exchange information with other good solutions, try some random perturbations, kill some not-so-good solutions and gradually evolve a suitable solution. There is no a priori reason why this process could not go on for a very long time before one solution becomes suitably good to enter consciousness. To the conscious mind, the sudden appearance of a suitable solution will seem to have arisen in an instant from nowhere or from a demon or from a god (Bateson, 1972).

Perhaps even human logic works this way. Consider the paradoxes of selfreference between levels beloved by Russell and Whitehead and by Godel. By a logical sequence of inferences, one is led in a vicious circle of contradiction. Consider the Liar Paradox:

All Cretans are liars.

I, a Cretan, am telling you so.

We predict that you can reason that this is an unsolvable paradox, but that you cannot experience the paradox consciously at any instant. Rather, you will experience a conscious train of reasoning that is 'locally' correct within the viscous circle. You answer true, false, true, etc. in succession; not all at once. Sentence 2 implies Sentence 1 must be false, so Cretans tell the truth. So Sentence 1 must be false. So Sentence 2 must be false. That is, Sentence 1 must be true. Then Sentence 2 is a lie. So Sentence 1 is true, And so on and on it goes. We, at least, must walk through this logical problem sequentially. However, our walk might be made more satisfying by several global hypotheses. First, that the problem as stated has no solution in single-level logic. Second, a solution has been suggested in which 'The Liar' is logically consistent in a multi-level system of logic (Barwise and Moss, 1996).

\section{Predictions on the unpredictable}

How would a creature that used the methods described here react to an observation that differed significantly from what its predictions caused it to expect?

First, we predict that the unexpected has a high probability of entering consciousness (as it may require conscious behavior to prevent or minimize harm). 
To mathematicians, scientists, and statisticians information is the unexpected. That is the essence of information theory. A limited consciousness needs the informative not the uninformative.

Second, we predict that the totally expected will have a low probability of expected occurrences entering consciousness (as we are already operating on the expectation of that occurrence). The attentional filter will allow into consciousness only that which it has not already unconsciously predicted to sufficient accuracy. This allows most activities to proceed unconsciously while devoting consciousness to the task for which it is best suiteddealing with the unexpected.

Third, we predict that there will be two distinct types of emotional responses to greet the unexpected. The most obvious is alarm. If strong enough, it will activate the 'fight or flight' function. But suppose we immediately discover that the unexpected is not threatening. Suppose it is purely benign. We might greet that realization with an experience of relief/problem averted - a kind of pleasure. That pleasurable experience, however it is elicited, should create the same emotional response.

\section{Is there direct evidence for unconscious modeling?}

We offer that the human brain contains models of its body. This is called 'proprioception' as in contrast with our modeling of the outside world that is called 'perception'. In fact it has two such models-one for sensor interpretation and one for effector control in the primary somatosensory cortex. This strip of brain material is located in the parietal lobe just behind (posterior to) the central fissure. The primary somatosensory cortex contains neurons that register the sense of touch. Similar to primary motor cortex, this strip of cortex is highly organized with specific regions representing each part of the body. Regions of the body that are capable of making finer discriminations of touch (palm of hand and mouth) have a larger area of representation than areas that are capable of gross discrimination only (such as the back or feet). Electrical stimulation of any of these specific regions results in the sensation of being touched on the represented body part. If the body structure changes, we must relearn the body model. A dramatic illustration is the gradual fading of the phantom limb experienced by many amputees. Other adjacent regions represent other body parts. So we form models or maps of the body.

But, what about the outside world? Do we form models or maps of this reality as well? Of course, the greatest evidence that we perceive a model world is that we perceive thing as being out there - remote from the brain doing the perceiving. The sensor signals we observe make no sense until we hypothesize that there is something that is 'not me.' 
Of course, the opposite also occurs and does so frequently. We do not consciously perceive things in the world that literally do not make sense. Darwin observed that many island people could not perceive the Beagle anchored far off shore, but clearly visible to him. Their experience did not include the possibility of such large ships. They had no trouble seeing the small boat he used to arrive on shore, however. The old saying 'Seeing is believing' is the exact opposite from the truth. We see what we unconsciously believe to be there and important to us. Believing is seeing!

We can sense something, carry out conscious behavior based on that sensed information, and still be unconscious of the sensory experience. In vision, this muchstudied phenomenon is called 'blindsight.' Patients are not conscious of visual perceptions they provably have (Caulfield, 1995)

\section{Do our predictions hold?}

\section{Predictions on Perception}

Do we perceive only one percept at any instant even when the stimulus is perfectly ambiguous? The ambiguous interpretation of Figure 1 offers the reader direct, personal experience of this phenomenon. Not only does this appear to be an artifact of the limited capabilities of consciousness, but also it appears to offer some insight into the control method used by our brains-unconscious and conscious in concert.

A personal case will illustrate the method clearly. Each day, I (HJC) drive well over a hundred miles. Sometimes I exceed the speed limit. On the rare occasions when the police catch me speeding, the experience is unpleasant. Both consciously (when I think about it consciously) and unconsciously (perhaps at all times), avoiding being caught speeding by a policeman is an important goal. Each time I see a police car, I slow down. That reaction has become purely habitual. I slow even when I am not speeding. What is interesting for this paper is that my unconscious attention mechanism resolves ambiguous scenes into the most important identification not the most probable one. I act on that and can correct my action later if subsequent observations remove the ambiguity. We accept the admonition of Antonio Damasio (1995) in his book Descartes 'Error.

Those who believe that little of the body state appears in consciousness under normal conditions may want to reconsider. It is true that we are not aware of every part of our body, all of the time, because representations of external events, through vision, hearing, or touch, as well as internally generated images, effectively distracted us from the ongoing, uninterruptible representation of the body. But the fact that our focus of attention is usually elsewhere, where it is 
most needed for adaptive behavior, does not mean the body representation is absent, as you can easily confirm whether sudden onset of pain or minor discomfort shifts the focus back to it. The background body sense is continuous, although one may hardly notice it, since it represents not a specific part of anything in the body but rather an overall state of most everything in it. Yet such an ongoing, unstoppable representation of the body state is what allows you to reply promptly to the specific question 'How do you feel?' with an answer that does relate to whether you feel fine or do not feel that well (152)

That by no means defeats our argument that we cannot attend all of the details at once. That is, no doubt, the reason we have a global sense of well being. We cannot possibly attend to all of the components unconsciously contributing to that global sense.

\section{Predictions on mental functioning}

Do inventors, scientists, poets musicians, saints, etc. experience a sudden enlightenment that solves a problem? That has been the near-universal testimony from Archimedes, Gautama, Moses, on. Moments of epiphany come both suddenly and unexpectedly. Many seek the experience for a long time before they have it. The prophet Mohammed spent years seeking a revelation. Jesus of Nazareth spent 40 days and nights of prayer and fasting in the wilderness, before an angel appeared to him. When the experience does come, even after much preparation, it seems to come unbidden. Every language has words to describe the experience: Eureka. Ah ha. Enlightenment. Epiphany. Revelation. Poets and musicians and painters speak of their 'demons' or 'muses.' That is an apt description, as they have little if any control over this wonderful experience. Some find, however, that prayer, fasting, drugs, chanting, dancing, meditation, or some other practice followed regularly does increase the frequency and/or intensity of the experience. The mathematician Poincare was so fascinated by the creative process that he spent years studying how he and others could give this type of event a greater chance of happening. We have examined the unconscious evolution of invention, inspiration, and revelation elsewhere (Caulfield, 1995 ) and propose not to repeat it in detail here. For these purposes, it is sufficient to note that the experience of a sudden useful insight is universal and consistent with our simple model. This is consistent with the sudden passing from the unconscious, which may have labored for years on the problem, to the conscious through the attentional filter. Until that moment, what was in the unconscious was not judged ready to bring to conscious attention.

Do individuals with multiple personality ever manifest more than one personality at a time? Certainly not. They have memories (sometimes consciously) 
of the other personality, but they are one person at any time. Philosophers, Buddhists, and others tell us that there is no continuing me, but I experience such a person. There is great survival value built into a system which considers that it has an ongoing identity and cares greatly about the future state of its being. Telling a consistent story to us about ourselves is a very difficult task that probably involves a special function as a storyteller (Gazzaniga, 1992).

In contemplating a paradox, are we ever conscious of the whole paradox at any instant? To our knowledge, this has not been studied. We cannot convince ourselves of own capabilities in this regard. Probably, a logic chain is inherently sequential, so a unified, instantaneous perception of the paradox seems unlikely. But we are certainly aware of it as a paradox as we march through the steps. The fact that we can contemplate a paradox without becoming paralyzed into inaction shows that mental processing quite different from that of a programmed Turing machine. Faced with 'The Liar Paradox' the Turing machine would never halt. Humans are aware of the paradox and simply accept it, albeit reluctantly for many mathematicians.

\section{Predictions on the unexpected}

Does the unexpected tend to draw our attention? Try it on your 'significant other' the next time he or she pretends to be listening yet is not consciously listening. Insert a nonsense sentence well out of context. 'The green cat ate my socks.' This unpredictable sentence typically would cause a reaction from the conscious state. Before, they were simply processing your words unconsciously. Or consider driving. You become conscious only when the unexpected occurs. Otherwise, you can drive for many miles unconscious of the process of driving, but consciously listening to the radio, talking on the cell phone, etc.

Remarkably, even the sensations usually associated with severe pain do not intrude on consciousness, it consciousness is concerned with something more urgent. An injury to your arm is unexpected. Suddenly your arm is in a state not predicted and considered undesirable. Typically, you become conscious of pain in that arm even if you were not consciously attending that arm. Your attentional filter has decided that you should be aware of that problem. However, in the case of an athletic event or combat, however, you may not be consciously aware of that problem. Your unconscious attention function may determine that your limited consciousness is better employed on other problems. It is routine for football players and soldiers to be unaware of gruesome injuries in the heat of battle. Survivors of lion attacks and similar catastrophes often report that they felt no pain at the time. Pain is the conscious experience of bodily injury.

Do expected events tend to fade from conscious awareness? This is one of the most common human experiences. As a youngster in Texas, I (HJC) would 
often visit a nearby town that had numerous oil fields. It reeked of SO2, H2S, and other nauseous gases, but the natives never noticed. The olfactory sensation provided no new information, and their attention process had no need let it to clutter their consciousness.

\section{Reprise}

It appears that the unconscious is modeling many parallel streams of sensation, and abstracting these into parallel streams of percepts. In some cases seeming contradictions appear, often observed to be accompanied by a 'bad feeling' (Sacks, 1995). However, conscious control requires a much-simplified task if it is to perform fast enough to be of value. So an attentional filter selects from the unconscious those streams of percepts, and those entailments of the internal models of body and world that most need conscious attention. This is a good design if the processor is very slow (relative to the events it must control). Since the process must perform an abduction on this information, and since abduction is necessarily non-local, the process requires a substrate spread out in space. Brains are the substrate of such processes. This model allows us to make and confirm numerous predictions about human conscious experience.

This is also a system that could have evolved from the control systems nonconscious animals. When the bodies and situations become too complex for a central control to operate in times needed to respond to the creature's situations in the world, two choices were available. It could develop parallel, largely independent control systems for different functions. Or it could develop a hierarchy of control centers with the lower levels dealing directly with the world and higher level center dealing with more compressed and selected summaries of the situation. Nature seldom makes these forced choices. More often, it takes both paths. Reflex loops are primitive examples of the parallel control path. The path of hierarchies dealing with less and less information at each level with the content of each higher level being selected by lower levels is the path we think led to consciousness.

\section{The human computer interface}

Many consequences flow from the fact that perception is unconscious before it is conscious. The more primitive unconscious parts of the brain add emotional color to the perception. The food seems delicious, the attractive other seems sexually desirable, the bear seems frightening. There is no 'immaculate perception.' Humans run the risk of misperceiving things in this way, so the best we can do is minimize the items that would cause emotional reactions. 
The most important consequence, however, is that we need to realize that humans have a very limited cognitive bandwidth. We select, through the attention process some of the available information and ignore the rest. The best HCI will provide context sensitive information content and update rate so as not to overwhelm the attention process of the human operator. Giving more information guarantees that some of what is presented will not be consciously perceived. But what will be ignored is unpredictable. Overloading is gambling. Presenting less information than the human can perceive does not give him the maximum information needed for his task.

\section{The way ahead}

Clearly, it seems to us, the trend in HCI to make better displays is not necessarily useful. It is far more important to develop Artificial Attention - a system between the Human and the Computer that selects what should be displayed at every moment in a way similar to the way your attention works.

1. It is normally focused on the task at hand.

2. When something is far out of expected value (The Artificial Attention system, like the human attention system, must be model based.), it normally comes to conscious attention.

3. The user can direct attention at will just as you will attend to your big toe on your left foot when you read this.

4. When these rules come into conflict, a referee must choose what to display (allow into consciousness) according to various rules. Soldiers injured in battle often do not feel pain during the battle. Their unconscious chooses not to attend to wounds when overall survival is at stake.

\section{Acknowledgements}

This paper is based in part on research was performed at Oak Ridge National Laboratory (ORNL), which is operated by UT-Battelle, LLC, for the U.S. Department of Energy under Contract No. DE-AC05-00OR22725. This paper is based in part on research performed at The NASA Center at Fisk University supported by the Army Research Office and the Electric Power Research Institute through a subcontract to Fisk University from Purdue University. We thank Mr. Roger Kisner (ORNL) and Dr. S. Alenka Brown-VanHoozer , Director, Center for Advanced Cognitive Technologies, BWXT-Y12, for their very helpful comments and corrections. 


\section{References}

Aiello, G. L. and Bach-Y-Rita, P. (2000) 'Nonsynaptically connected neural nets' in Proceedings - European Symposium on Artificial Neural Networks, Bruges BELGIUM, 26-28, pp. 425-430.

Barwise, J. and Moss, L., 1996, Vicious Circles: On the Mathematics ofNon-wellfounded

Phenomena. Stanford, CA: Center for the Study of Language and Information.

Bateson, G. (1972) Steps to an Ecology of Mind, Chicago: University of Chicago Press,

Caulfield, H. J. (1995) 'The computer unconscious,' Kybemetes 24, 46-52.

Creutzfeldt, D. (1979) Neurophysiological mechanisms and consciousness. Ciba

Foundation Sympposium, 1979:69 217-33.

Damasio, A. R, (1999) The Feeling of What Happens, Harcourt, Brace and Company, New York.

Damasio, A. R., (1995) Descartes' Error. Emotion, Reason, and the Human Brain, Avon Books.

Dilts, R, (1983) Roots of Neuro-Linguistic Programming, Cupertino CA: Meta Publications, 1983, pp. 17-18

Fodor, J. (2000) The Mind Doesn't Work That Way: The Scope and Limits of Computational Psychology (Representation and Mind), Cambridge MA: MIT Press.

Freeman, W. J. (1999) How Brains Make Up Their Minds, Weidenfeld and Nicholson (London), 1999, pp. 67-193.

Gazzaniga, Michael S., 1992, Nature's Mind: The Biological Roots of Thinking, Emotions, Sexuality, Language, and Intelligence. New York: Basic Books.

Kernel, S. W. (2001) Does Incomputable Mean not Engineerable? In Smart Engineering System Design: Neural Networks, Fuzzy Logic, Evolutionary Programming, Data Mining and Complex Systems, Vol. 11, New York: ASME Press.

Korsybski, A. (2000) Science and Sanity. 5th ed. second printing. Brooklyn NY: Institute of General Semantics, p. lxii.

Kovacs, T. V. Papathomas, and M. Yang, A. Feher (1996) When the brain changes its mind: Interocular grouping during binocular rivalry. Proceedings of the Naional Academy of Science USA, 93: 15508-15511, 1996.

Libet, B., Freeman, A. and Sutherland, K. (eds.) (1999). The Volitional Brain Towards a Neuroscience of Free Will. Thorverton, UK: Imprint Academic, 1999. Series title: Journal of Consciousness Studies.

Rosen, R., (1991) Life Itself: A Comprehensive Inquiry into the Nature, Origin, and Fabrication of Life, New York: Columbia University Press, pp. 241-242.

Sacks, O. (1995) An Anthropologist on Mars, Vintage Books, New York. pp. 269-271. 


$$
\partial
$$

